\title{
Changes in motor cortex excitability associated with temporal repetitive transcranial magnetic stimulation in tinnitus: hints for cross-modal plasticity?
}

Martin Schecklmann ${ }^{1 *}$, Michael Landgrebe ${ }^{4}$, Tobias Kleinjung ${ }^{3}$, Elmar Frank1, Philipp G Sand ${ }^{1}$, Rainer Rupprecht ${ }^{1}$, Peter Eichhammer', Göran Hajak² and Berthold Langguth ${ }^{1}$

\begin{abstract}
Background: Motor cortex excitability was found to be changed after repetitive transcranial magnetic stimulation (rTMS) of the temporal cortex highlighting the occurrence of cross-modal plasticity in non-invasive brain stimulation. Here, we investigated the effects of temporal low-frequency rTMS on motor cortex plasticity in a large sample of tinnitus patients. In 116 patients with chronic tinnitus different parameters of cortical excitability were assessed before and after ten rTMS treatment sessions. Patients received one of three different protocols all including $1 \mathrm{~Hz}$ rTMS over the left temporal cortex. Treatment response was defined as improvement by at least five points in the tinnitus questionnaire (TQ). Variables of interest were resting motor threshold (RMT), short-interval intra-cortical inhibition (SICI), intracortical facilitation (ICF), and cortical silent period (CSP).

Results: After rTMS treatment RMT was decreased by about 1\% of stimulator output near-significantly in the whole group of patients. $\mathrm{SICl}$ was associated with significant changes with respect to treatment response. The group of treatment responders showed a decrease of $\mathrm{SICl}$ over the course of treatment, the group of non-responders the reverse pattern.

Conclusions: Minor RMT changes during rTMS treatment do not necessarily suggest the need for systematic re-examination of the RMT for safety and efficacy issues. Treatment response to rTMS was shown to be related to changes in $\mathrm{SICl}$ that might reflect modulation of GABAergic mechanisms directly or indirectly related to rTMS treatment effects.
\end{abstract}

Keywords: Transcranial magnetic stimulation, Motor cortex excitability, Motor cortex plasticity, Tinnitus, Cross-modal plasticity, Cortical silent period, Motor threshold, Intracortical inhibition, Intracortical facilitation

\section{Background}

Tinnitus is associated with neural changes in both the auditory pathway and in non-auditory brain areas [1]. Tinnitus related changes of activity and connectivity in frontal, temporal, parietal, and limbic areas e.g. [2,3] seem to reflect pathologically altered brain networks $[4,5]$. Based on these findings repetitive transcranial magnetic stimulation (rTMS) was introduced as a treatment approach in

\footnotetext{
* Correspondence: martin.schecklmann@medbo.de

'Department of Psychiatry and Psychotherapy, University Regensburg,

Universitätsstraße 84, 93053 Regensburg, Germany

Full list of author information is available at the end of the article
}

tinnitus [6]. However, it turned out that the treatment effects are moderate and associated with high inter-individual variability which raises the need for indicators for effective therapy. Clinical trials of rTMS in tinnitus use typically stimulation of one or both temporal/auditory or temporoparietal cortices. Recently these protocols were extended by additional stimulation of non-auditory areas such as the frontal cortex to target tinnitus-specific networks more effectively $[7,8]$.

Repetitive transcranial magnetic stimulation (rTMS) as a therapeutic intervention consists of non-invasive repeated stimulation of neocortical areas for hundreds of 
times per daily sessions via principles of electromagnetic induction. The TMS coil is placed on the subjects' head over the target area and short-lasting high-intense currents in the coil produce a strong magnetic field (up to $1.5 \mathrm{~T}$ ), which passes largely undistorted through the scull and induces neuronal depolarisations in the underlying cortical area. rTMS leads to long-term depression- or potentiationlike neuroplastic changes [9]. Low-frequency $1 \mathrm{~Hz}$ rTMS of motor cortex induces long-term depression as indicated by motor cortex excitability parameters [10]. Effects take place in the directly stimulated cortical area and also in functionally connected remote areas, presumably via corticocortical connectivity [11]. For example, stimulation of the dorso-lateral prefrontal cortex is associated with blood flow changes in the anterior cingulate cortex $[11,12]$ or dopamine release in the caudate nucleus [13].

In addition TMS can also be used as a diagnostic tool for the assessment of motor cortex excitability by quantifying contractions of peripheral muscles induced by stimulation of the corresponding motor cortex representation. Assessment of motor cortex excitability in a longitudinal study design before and after a specific therapeutic intervention (e.g. multiple sessions of rTMS) enables to describe treatment-related neuroplastic changes. It has been shown, that rTMS over the temporal cortex can induce changes in motor cortex excitability. In a sham-controlled study 27 healthy subjects showed decreased motor evoked potential amplitudes and delayed cortical silent period (CSP) after five days of $1 \mathrm{~Hz}$ rTMS of the right temporal cortex [14]. Furthermore, these changes were accompanied by a decrease in glucose metabolism in the stimulated temporal cortex and an increase in cingulate and frontal areas but also in motor cortex. Five days of active low-frequency rTMS over auditory cortex lead to prolongation of the CSP in 18 healthy subjects [15], whereas sham rTMS had not such effects. Clinical benefit of the same protocol in ten patients with tinnitus was positively correlated with changes in short-interval intra-cortical inhibition (SICI), intracortical facilitation (ICF), and CSP [16].

In conclusion, these studies highlight the existence of cross-modal plasticity in rTMS studies in tinnitus or with temporal stimulation. Evidence for functional connectivity between temporal and motor cortex comes from neurophysiologic and brain metabolism studies $[17,18]$. In tinnitus, the interaction between the sensorimotoric and the auditory system is well established and clinically reflected by somatosensoric modulation of the tinnitus percept [19]. Here, we aimed to investigate the effects of temporal rTMS on different motor cortex excitability parameters in the biggest investigated tinnitus sample so far by means of a retrospective analysis of data obtained in the context of different clinical trials. As only one study in tinnitus with a small sample size was done so far we especially focused on the association of clinical response with change in excitability in the quest for a non-auditory neurophysiological indicator for effective therapy.

\section{Methods}

\section{Subjects}

All participants gave written informed consent after a comprehensive explanation of the procedures. All studies whose data contributed to this analysis were approved by the Ethics Committee at the University of Regensburg. All experiments were conducted in accordance with the last revision of the Declaration of Helsinki.

Measurements of cortical excitability were performed in 116 patients (84 (72.4\%) males; $49.2 \pm 12.5$ (21-83) years) with chronic tinnitus (duration $90 \pm 94$ (2-476) months). 28 (25.2\%) out of 111 patients (data of 5 patients not available) reported a purely left-sided, 21 (18.9\%) a purely right-sided tinnitus, and 62 (55.9\%) patients described their tinnitus as bilateral or originating within the head. Tinnitus distress was assessed by the German version [20] of the Tinnitus questionnaire (TQ) [21]; TQ baseline scores ranged from 3 to $79(41 \pm 18)$. Patients suffering from Meniere's disease, presenting conductive hearing loss or displaying hints of objective tinnitus were not included. 62 patients underwent a complete otologic and audiologic examination including pure tone audiometry, tympanometry, stapedius reflex tests, and otoscopy. Mean hearing level of the audiogram (bilateral hearing thresholds at $0.125,0.25,0.5,1,2,3,4,6,8 \mathrm{kHz}$ ) was $17 \pm 13(0-61 \mathrm{~dB}$ HL) . Only patients were included that were eligible for rTMS treatment. Thus, patients with cardiac pacemakers, history of seizures, suspected diagnosis of organic brain damage or any other severe somatic, neurologic, or psychiatric diagnosis were not included.

\section{Procedures}

The therapeutic intervention consisted of 10 rTMS sessions on consecutive weekdays. Treatment effects were evaluated by changes in TQ between the first (day 1) and the last day of treatment (day 12). Motor cortex excitability was examined on the first day before treatment and on the last day after rTMS. We analyzed longitudinal data of 116 patients participating in three different treatments studies [7,22]. Patients received one out of three different active stimulation protocols (2000 stimuli over auditory cortex with $1 \mathrm{~Hz}: \mathrm{n}=68 ; 4000$ stimuli over auditory cortex with $1 \mathrm{~Hz}: \mathrm{n}=26 ; 2000$ stimuli over left frontal cortex with $20 \mathrm{~Hz}$ followed by 2000 stimuli over auditory cortex with $1 \mathrm{~Hz}: \mathrm{n}=22$ ). Stimulation was set to $110 \%$ of the individual resting motor threshold. Localization of the stimulated areas was either done with a neuronavigational system or by using a standard procedure based on the 10-20 system [23]. Recent analyses did not reveal clinically relevant differences in treatment efficacy depending 
on the used method for coil positioning [7]. In detail, each patient was treated with one protocol lasting ten days and one kind of localization that means that there were sub-groups with different treatments. Data were recorded from 2004 to 2009. All measurements were performed by the same staff which was experienced in the used methods.

For measurement of cortical excitability, participants were seated in a reclining chair. TMS was delivered by two Magstim 200 stimulators (Magstim Co., UK) connected via a Bistim module to a figure-of-eight coil (double-circular-70-mm coil). The coil was held tangential to the skull and with the handle pointing backwards and about $45^{\circ}$ away from the midline. The optimal coil position for stimulation was defined as the position above the left motor cortex for eliciting MEP of maximal amplitude in abductor digiti minimi muscle with a slightly supra-threshold stimulus. Once this position was found, it was marked on a scientific head cap and the coil was held in this position by the investigator.

Motor evoked potentials (MEPs) of the abductor digiti minimi of the right hand were recorded with surface electrodes. The analogue signal was registered, bandpass filtered between $20 \mathrm{~Hz}$ and $10 \mathrm{kHz}$, then digitised at a frequency of $5 \mathrm{kHz}$ and analysed off-line. Resting motor threshold (RMT) was determined as the lowest stimulation intensity that evoked in at least four out of eight consecutive trials a MEP of at least $50 \mu \mathrm{V}$ in the resting abductor digiti minimi [24]. Active motor threshold was defined as the lowest stimulation intensity that evoked in at least four out of eight consecutive trials a $\mathrm{MEP}$ of at least $250 \mu \mathrm{V}$ during isometric contraction of the abductor digiti minimi at about $20 \%$ of maximum voluntary contraction. A constant level of voluntary contraction was maintained by audiovisual feedback of the electromyographic (EMG) activity. MEP amplitudes were measured peak-to-peak.

Short-interval intra-cortical inhibition (SICI) and intra-cortical facilitation (ICF) were measured with a paired-pulse TMS protocol [25]. The intensity of the first (conditioning) stimulus was set at $90 \%$ of the active motor threshold. The second stimulus was delivered at an intensity that produced MEPs of about $1 \mathrm{mV}$ in the resting abductor digiti minimi. Interstimulus intervals (ISIs) were $2 \mathrm{~ms}$ and $15 \mathrm{~ms}$ to measure short-interval intra-cortical inhibition (reduction of amplitude) and intracortical facilitation (increase of amplitude), respectively [25]. The conditioned stimuli and the control condition (test pulse alone) were each tested 10 times in a random order (inter-sweep-interval: $4 \mathrm{~s}$ ). The effect of conditioning stimuli on MEP amplitude at each ISI was determined as the ratio of the average amplitude of conditioned MEP (cMEP) to the average amplitude of unconditioned test MEP (uMEP).
Cortical silent period was measured in 10 trials (stimulus intensity: $150 \%$ resting motor threshold; inter-sweepinterval: $5 \mathrm{~s}$ ) in the moderately active abductor digiti minimi muscle on the non-rectified recording of every individual sweep and then averaged [26]. Participants were instructed to contract this muscle at $30 \%$ maximum strength. The onset of the cortical silent period was defined as the end of the MEP when activity dropped consistently below pre-stimulus EMG level. The end of the cortical silent period was defined as first reappearance of voluntary EMG activity. In conclusion, TMS variables of interest were resting motor threshold (RMT), cMEP/uMEP ratio of $2 \mathrm{~ms}$ and $15 \mathrm{~ms}$ interstimulus intervals (SICI and ICF, respectively), and cortical silent period (CSP).

\section{Statistics}

Statistics are based on retrospective analysis. We were interested if changes of motor cortex excitability were related to rTMS treatment per se and to rTMS induced clinical response. Thus, we did an analysis of variance with treatment as within-subjects factor (first vs. last day of treatment) and treatment response as betweensubjects factor (responder vs. non-responder). Treatment response was defined as change in TQ of at least 5 points $[27,28]$. If an ANOVA revealed significant results, we performed post-hoc t-tests for comparison of responders and non-responders before and after rTMS and for the changes in both groups over time. We were interested in effects of rTMS protocols as potential confounder and repeated these ANOVAs with rTMS protocol as covariate (2000 stimuli temporal vs. 4000 stimuli temporal vs. 2000 stimuli frontal and 2000 temporal) for the significant effects.

As we had four variables of interest (RTM, SICI, ICF, and CSP), significance threshold was set to a Bonferroni corrected level $(0.05 / 4=0.0125)$. For post-hoc tests significance threshold was set to 5\%. Statistical analyses were performed with SPSS 18.0.0 (SPSS, USA). As the focus of this analysis were rTMS induced effects on cortical excitability we abstain from reporting treatment efficacy data which have already been published elsewhere $[7,22,29,30]$.

\section{Results}

Firstly, rTMS resulted in a near significant decrease in RMT (reduction by 1\% stimulator output) in the whole study population (Table 1). Other parameters of motor cortex excitability remained unchanged. We did not find significant interaction effects of "rTMS protocol" with change in RMT, SICI, ICF, and CSP.

We found no significant effects of treatment (pre vs. post rTMS). For RMT there was a tendency towards a reduction (of about $1 \%$ stimulator output; $\mathrm{p}=0.024$ ) but 
Table 1 Descriptive and statistical data of changes in motor cortex excitability in dependence from rTMS intervention and treatment response

\begin{tabular}{|c|c|c|c|c|c|c|}
\hline & & pre rTMS & post rTMS & $\begin{array}{c}\text { Main effect } \\
\text { treatment }\end{array}$ & $\begin{array}{l}\text { Main effect } \\
\text { response }\end{array}$ & $\begin{array}{c}\text { Interaction } \\
\text { effect }\end{array}$ \\
\hline \multirow[t]{3}{*}{ resting motor threshold } & \multirow{2}{*}{$\begin{array}{c}\text { non- } \\
\text { responder }\end{array}$} & \multirow[t]{2}{*}{$42.6 \pm 8.2$} & \multirow[t]{2}{*}{$41.5 \pm 8.5$} & $F=52.679$ & $F=0.062$ & $F=0.268$ \\
\hline & & & & $\mathrm{df}=1,114$ & $d f=1,114$ & $\mathrm{df}=1,114$ \\
\hline & responder & $42.8 \pm 7.8$ & $42.0 \pm 8.0$ & $p=0.024^{+}$ & $p=0.805$ & $p=0.606$ \\
\hline \multirow{3}{*}{$\begin{array}{c}\text { short-interval intra-cortical } \\
\text { inhibition }\end{array}$} & \multirow{2}{*}{$\begin{array}{l}\text { non- } \\
\text { responder }\end{array}$} & \multirow[t]{2}{*}{$0.58 \pm 0.51$} & \multirow[t]{2}{*}{$0.47 \pm 0.28$} & $F=0.319$ & $F=2.323$ & $F=6.447$ \\
\hline & & & & $\mathrm{df}=1,114$ & $\mathrm{df}=1,114$ & $\mathrm{df}=1,114$ \\
\hline & responder & $0.41 \pm 0.29$ & $0.47 \pm 0.33$ & $p=0.574$ & $p=0.130$ & $p=0.012^{*}$ \\
\hline \multirow[t]{3}{*}{ intra-cortical facilitation } & \multirow{2}{*}{$\begin{array}{l}\text { non- } \\
\text { responder }\end{array}$} & \multirow[t]{2}{*}{$1.68 \pm 1.24$} & \multirow[t]{2}{*}{$1.43 \pm 0.74$} & $F=3.079$ & $F=3.474$ & $F=2.823$ \\
\hline & & & & $d f=1,114$ & $d f=1,114$ & $d f=1,114$ \\
\hline & responder & $1.32 \pm 0.39$ & $1.32 \pm 0.36$ & $p=0.082$ & $p=0.065$ & $p=0.096$ \\
\hline \multirow[t]{3}{*}{ cortical silent period } & \multirow{2}{*}{$\begin{array}{l}\text { non- } \\
\text { responder }\end{array}$} & \multirow{2}{*}{$\begin{array}{c}0.108 \pm \\
0.040\end{array}$} & \multirow{2}{*}{$\begin{array}{c}0.107 \pm \\
0.044\end{array}$} & $F=0.080$ & $F=1.818$ & $F=0.179$ \\
\hline & & & & $d f=1,114$ & $d f=1,114$ & $d f=1,114$ \\
\hline & responder & $\begin{array}{c}0.118 \pm \\
0.065\end{array}$ & $\begin{array}{c}0.123 \pm \\
0.104\end{array}$ & $p=0.778$ & $p=0.180$ & $p=0.673$ \\
\hline
\end{tabular}

*significant at a Bonferroni corrected threshold with p-values displayed uncorrected; ${ }^{+}$near significant at a Bonferroni corrected threshold with p-values displayed uncorrected.

this effect did not reach the Bonferroni-corrected significance level. There was no main effect of group (responder vs. non-responder) either. With respect to the group $\mathrm{x}$ treatment interaction there was a significant interaction effect between the change in SICI and the clinical response to rTMS $(\mathrm{p}=0.012$; Table 1 , Figure 1$)$. As post-hoc t-tests indicate, responders showed a reduction of SICI $(\mathrm{t}=2.327 ; \mathrm{df}=56 ; \mathrm{p}=0.024)$ and nonresponders an increase in $\operatorname{SICI}(\mathrm{t}=1.737 ; \mathrm{df}=58 ; \mathrm{p}=$ $0.088)$ due to the rTMS intervention. Please note that reduction in SICI is mirrored by a numerical increase of the amplitude ratio between the conditioned stimulus and the test stimulus. Responders significantly differed in their SICI from non-responders before treatment $(t=2.282$;

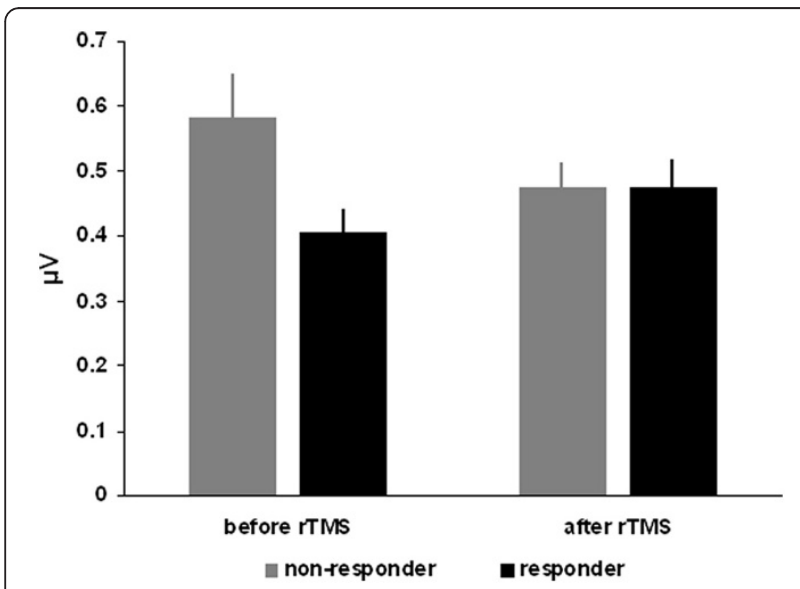

Figure 1 Raw data (mean \pm se) of the short-interval intracortical inhibition (SICl) for the significant interaction effect of the analysis of variance with the factors rTMS treatment and treatment response. $\mathrm{df}=114 ; \mathrm{p}=0.024)$ whereas there was no difference at the end of treatment $(t=0.002 ; d f=114 ; p=0.999)$. For the other investigated parameter (RMT, ICF, CSP) the interaction group $\mathrm{x}$ treatment was not significant. ANOVAs with rTMS protocol as covariate affirmed the significant findings for RMT ( $\mathrm{p}=0.091)$ and SICI $(\mathrm{p}=0.013)$ indicating no influence of the rTMS protocol. Detailed statistic and descriptive data are given in Table 1.

\section{Discussion}

Treatment of 10 days of rTMS over auditory cortex in 116 patients with chronic tinnitus resulted in a near significant, but very small reduction of RMT ( $1 \%$ stimulator output) for the whole group. Since stimulation intensity in most rTMS treatment studies is adjusted to the resting motor threshold (RMT) several earlier studies investigated whether rTMS treatment changes the RMT [5,14-16,31-42]. Findings in these studies are not entirely consistent and it also remains unclear whether observed fluctuations of RMT during the course of rTMS treatment rather reflect variations of cortical excitability or rather an inherent inaccuracy of the measurement procedure of the RMT [43]. Despite these uncertainties there are recommendations to re-measure the RMT over the course of treatment and to adjust the stimulation intensity accordingly [5]. However, the mean reduction by about $1 \%$ of the stimulator output in our sample rather suggests that the induced changes in RMT are negligible. Changes in cortical excitability would be meaningful if RMT were shown to be reliable and if stimulation intensity was falling above safe intensity or below effective targeting the brain due to changes of RMT. 
Treatment responders as identified by a 5-point reduction in the TQ showed increased SICI at the beginning of the treatment and a reduction over the course of the treatment. Non-responders showed the reverse pattern. Because of the lack of a placebo group we cannot definitively conclude that the observed reduction of SICI during treatment in the responder-group is related to successful rTMS treatment. Theoretically the SICI change could also reflect tinnitus reduction alone, independently of the kind of intervention. However the fact that the responder and non-responder group also differed in their baseline values of SICI makes the latter explanation extremely unlikely.

A relationship between treatment response and a reduction of SICI has not yet been reported before. The discrepancy to previous results may be related to statistical power since the here presented data are from the biggest sample so far. Two studies in healthy controls found no effects of temporal rTMS on SICI $[14,15]$; in a small sample of tinnitus patients low frequency temporal rTMS increased SICI [16] and also high frequency prefrontal rTMS in patients with major depression increased SICI [31]. However, authors stimulated the left dorso-lateral prefrontal cortex with $20 \mathrm{~Hz}$. We used three different treatments all including temporal $1 \mathrm{~Hz}$ stimulation of the auditory cortex with one treatment including a $20 \mathrm{~Hz}$ stimulation of left dorso-lateral prefrontal cortex before the temporal stimulation. We did not find any treatment specific effects. One could speculate that opposite effects on SICI after low frequency (decrease of SICI in the present study) and high-frequency rTMS [increase of SICI in 31] may reflect the well-established frequency-dependency of the direction of rTMS treatment effects [10]. However, it is unclear, whether the findings from motor cortex can be directly transferred to nonmotor areas since the exact mechanism of the observed cross-modal interaction still remains to be elucidated.

Nevertheless, our findings of altered SICI add further evidence to the notion of cross-modal plasticity of motor cortex in context of stimulation of non-motor areas. Considered mechanisms as indicated by studies in synaesthesia and sensory deprivation (e.g. deafness or blindness) may take place via multi-sensory association areas, via direct cortico-cortical connections, or via subcortical interplay of the corresponding sensory systems at the thalamic level [44-47]. In tinnitus and other neuropsychiatric disorders, the model of thalamocortical dysrhythmia considers altered thalamic activity due to deprived afferent input as the core of the pathology. This altered thalamic activity is generating altered cortical activity which in turn induces symptoms such as phantom perceptions [48]. Based on this concept one could assume that the observed effects on motor cortex excitability in treatment responders are mediated via rTMS induced modulation of thalamic activity. The exact mechanism which underlies the observed cross-modal plasticity effect in this study might be best evaluated by future connectivity studies. Dynamic causal modelling would enable the modelling of the direction of information flow of assumed network hubs. Such studies could elucidate, whether our findings may reflect region-specific changes mediated via specific cross-modal pathways or rather global changes as an unspecific response to local stimulation.

Beside anatomical considerations of cross-modal plasticity, our findings can also be discussed in the context of trans-synaptic chemical signalling. Both antidepressant effects [31] and tinnitus reduction [49], are mediated by the inhibitory-acting $\gamma$-aminobutyric acid (GABA). GABA is also involved in motor cortex excitability and plasticity for an overview [50,51]. Especially SICI is mediated by GABAergic interneurons within the primary motor cortex [50,52]. For tinnitus, evidence for GABAergic involvement comes from pharmacological treatments [for an overview 49], animal models [53], and genetic analyses e.g., [54]. Recent studies in animal models of tinnitus identified deficient inhibitory function in input-deprived auditory regions [53]. If low frequency rTMS reduces tinnitus by renormalizing inhibitory function in the auditory cortex, the observed reduction of SICI in treatment responders could reflect the subsequent reduction of a compensatory global inhibitory effort. Following this reasoning the increased baseline SICI may then reflect reduced inhibitory tone in the auditory system. It seems reasonable that rTMS can only exert an effect on tinnitus, if the inhibitory function in the auditory system is altered. In this study we found no effects for other excitability measures. Since RMT, ICF and CSP are rather modulated by other neurotransmitters and -receptors, our findings highlight the role of GABAA mediated neurotansmission in rTMS treatment effects in tinnitus [41,42]. We are well aware that this interpretation is highly speculative and needs to be confirmed by further studies, before further conclusions can be drawn.

On a functional level auditory-motor connectivity might be related to functions of speech or music perception. The motor theory of speech perception links vocal tract gestures to the perception of speech [55]. Beat induction and rhythm perception helps to identify regular patterns in music with the aim to get synchronised by clapping, dancing, or singing [56]. Electrophysiological markers of this transfer may be grounded in the existence of rhythmic oscillatory activity of mu or alpha-like frequencies [57]. Based on these considerations the anatomical, neurotransmitter, and functional association of treatment response to temporal stimulation in tinnitus with SICI highlights the role of auditory-motor connectivity in rTMS treatment of tinnitus. Future clinical approaches might be the identification of responders of 
daily rTMS based on measurements of SICI changes after single rTMS sessions.

\section{Conclusions}

The counterpoint to the big sample size are the missing control groups and the retrospective analysis. Therefore further prospective studies including control groups are needed before firm conclusions about the specifity of the observed effects for rTMS treatment of tinnitus can be drawn. Moreover consecutive, daily measurements would enable to better characterise the time course of changes in excitability.

Nevertheless the presented data add to the literature that rTMS of non-motor areas can induce changes in motor cortex excitability. RMT changes during rTMS treatment seem to be negligible and do not affirm the potential need for re-evaluation of the stimulation intensity during treatment for efficacy and safety issues. SICI changes might reflect modulation of GABAergic mechanisms directly or indirectly related to rTMS treatment effects.

\section{Competing interests}

No author has to declare competing interest except the receipt of thirdparty funds from the Tinnitus Research Initiative for the conduction of clinical trials of rTMS in tinnitus.

\section{Authors' contributions}

MS, ML, TK, EF, PGS, PE, GH, BL have made substantial contributions to conception and design, or acquisition of data, or analysis and interpretation of data. All authors have been involved in drafting the manuscript or revising it critically for important intellectual content. MS, TK, RR, and BL have given final approval of the version to be published. All authors agree to be accountable for all aspects of the work in ensuring that questions related to the accuracy or integrity of any part of the work are appropriately investigated and resolved. All authors read and approved the final manuscript.

\section{Acknowledgments}

The authors wish to thank Sandra Pfluegl and Helene Niebling for technical assistance.

\section{Author details \\ 'Department of Psychiatry and Psychotherapy, University Regensburg, Universitätsstraße 84, 93053 Regensburg, Germany. ${ }^{2}$ Department of Psychiatry, Psychosomatic Medicine and Psychotherapy, Social Foundation Bamberg, Bamberg, Germany. ${ }^{3}$ Department of Otorhinolaryngology, University of Zurich, Zurich, Switzerland. ${ }^{4}$ Department of Psychiatry, Psychosomatics and Psychotherapy, kbo-Lech-Mangfall-Klinik, Agatharied, Germany.}

Received: 17 December 2013 Accepted: 21 May 2014 Published: 4 June 2014

\section{References}

1. Adjamian P, Sereda M, Hall DA: The mechanisms of tinnitus: perspectives from human functional neuroimaging. Hear Res 2009, 253(1-2):15-31.

2. Maudoux A, Lefebvre P, Cabay JE, Demertzi A, Vanhaudenhuyse A Laureys S, Soddu A: Auditory resting-state network connectivity in tinnitus: a functional MRI study. PLoS One 2012, 7(5):e36222.

3. Schlee W, Hartmann T, Langguth B, Weisz N: Abnormal resting-state cortical coupling in chronic tinnitus. BMC Neurosci 2009, 10:11.

4. De Ridder D, Elgoyhen AB, Romo R, Langguth B: Phantom percepts: tinnitus and pain as persisting aversive memory networks. Proc Natl Acad Sci U S A 2011, 108(20):8075-8080.
5. Zarkowski P, Navarro R, Pavlicova M, George MS, Avery D: The effect of daily prefrontal repetitive transcranial magnetic stimulation over several weeks on resting motor threshold. Brain Stimul 2009, 2(3):163-167.

6. Langguth B, Schecklmann M, Lehner A, Landgrebe M, Poeppl TB, Kreuzer PM, Schlee W, Weisz N, Vanneste S, De Ridder D: Neuroimaging and neuromodulation: complementary approaches for identifying the neuronal correlates of tinnitus. Front Syst Neurosci 2012, 6:15.

7. Langguth $B$, Landgrebe M, Frank E, Schecklmann M, Sand PG, Vielsmeier V, Hajak G, Kleinjung T: Efficacy of different protocols of transcranial magnetic stimulation for the treatment of tinnitus: pooled analysis of two randomized controlled studies. World J Biol Psychiatry 2012, 15(4):276-285.

8. Lehner A, Schecklmann M, Kreuzer PM, Poeppl TB, Rupprecht R, Langguth B: Comparing single-site with multisite rTMS for the treatment of chronic tinnitus - clinical effects and neuroscientific insights: study protocol for a randomized controlled trial. Trials 2013, 14:269.

9. Wassermann EM, Zimmermann T: Transcranial magnetic brain stimulation: therapeutic promises and scientific gaps. Pharmaco/ Ther 2012, 133(1):98-107.

10. Fitzgerald PB, Fountain S, Daskalakis ZJ: A comprehensive review of the effects of rTMS on motor cortical excitability and inhibition. Clin Neurophysiol 2006, 117(12):2584-2596.

11. Paus T, Castro-Alamancos MA, Petrides M: Cortico-cortical connectivity of the human mid-dorsolateral frontal cortex and its modulation by repetitive transcranial magnetic stimulation. Eur J Neurosci 2001, 14(8):1405-1411.

12. Esslinger C, Schuler N, Sauer C, Gass D, Mier D, Braun U, Ochs E, Schulze TG, Rietschel M, Kirsch P, Meyer-Lindenberg A: Induction and quantification of prefrontal cortical network plasticity using $5 \mathrm{~Hz}$ rTMS and fMRI. Hum Brain Mapp 2012, 35(1):140-151.

13. Strafella AP, Paus T, Barrett J, Dagher A: Repetitive transcranial magnetic stimulation of the human prefrontal cortex induces dopamine release in the caudate nucleus. J Neurosci 2001, 21(15):RC157.

14. Lee M, Kim SE, Kim WS, Han J, Kim HJ, Kim BS, Kim JY, Hong SB, Kim BG, Lee HW: Cortico-cortical modulation induced by $1-\mathrm{Hz}$ repetitive transcranial magnetic stimulation of the temporal cortex. J Clin Neurol 2013, 9(2):75-82.

15. Eichhammer $P$, Kleinjung $T$, Landgrebe M, Hajak G, Langguth B: TMS for treatment of chronic tinnitus: neurobiological effects. Prog Brain Res 2007, 166:369-375.

16. Langguth $B$, Kleinjung $T$, Marienhagen J, Binder $H$, Sand PG, Hajak G, Eichhammer P: Transcranial magnetic stimulation for the treatment of tinnitus: effects on cortical excitability. BMC Neurosci 2007, 8:45.

17. Graziano MS, Reiss LA, Gross CG: A neuronal representation of the location of nearby sounds. Nature 1999, 397(6718):428-430.

18. Speer AM, Willis MW, Herscovitch P, Daube-Witherspoon M, Shelton JR, Benson BE, Post RM, Wassermann EM: Intensity-dependent regional cerebral blood flow during $1-\mathrm{Hz}$ repetitive transcranial magnetic stimulation (rTMS) in healthy volunteers studied with $\mathrm{H} 2150$ positron emission tomography: II. Effects of prefrontal cortex rTMS. Biol Psychiatry 2003, 54(8):826-832.

19. Koehler SD, Shore SE: Stimulus timing-dependent plasticity in dorsal cochlear nucleus is altered in tinnitus. J Neurosci 2013, 33(50):19647-19656.

20. Göbel G, Hiller W: The tinnitus questionnaire. A standard instrument for grading the degree of tinnitus. Results of a multicenter study with the tinnitus questionnaire. HNO 1994, 42(3):166-172.

21. Hallam RS, Jakes SC, Hinchcliffe R: Cognitive variables in tinnitus annoyance. Br J Clin Psychol 1988, 27(Pt 3):213-222.

22. Lehner A, Schecklmann M, Landgrebe M, Kreuzer PM, Poeppl TB, Frank E, Vielsmeier V, Kleinjung T, Rupprecht R, Langguth B: Predictors for rTMS response in chronic tinnitus. Front Syst Neurosci 2012, 6:11

23. Langguth B, Zowe M, Landgrebe M, Sand P, Kleinjung T, Binder H, Hajak G, Eichhammer P: Transcranial magnetic stimulation for the treatment of tinnitus: a new coil positioning method and first results. Brain Topogr 2006, 18(4):241-247.

24. Rossini PM, Barker AT, Berardelli A, Caramia MD, Caruso G, Cracco RQ, Dimitrijevic MR, Hallett M, Katayama Y, Lucking CH, Maertens de Noordhout AL, Marsden CD, Murray NMF, Rothwell JC, Swash M, Tomberg C: Non-invasive electrical and magnetic stimulation of the brain, spinal cord and roots: basic principles and procedures for routine clinical application. Report of an IFCN committee. Electroencephalogr Clin Neurophysiol 1994, 91(2):79-92. 
25. Kujirai T, Caramia MD, Rothwell JC, Day BL, Thompson PD, Ferbert A, Wroe S, Asselman P, Marsden CD: Corticocortical inhibition in human motor cortex. J Physiol 1993, 471:501-519.

26. Wolters A, Ziemann U, Benecke R: The cortical silent period. In The Oxford Handbook of Transcranial Stimulation. Edited by Wasserman EM, Epstein CM, Ziemann U, Walsh V, Paus T, Lisanby SH. Oxford: University Press; 2008.

27. Kleinjung T, Steffens T, Sand P, Murthum T, Hajak G, Strutz J, Langguth B, Eichhammer $P$ : Which tinnitus patients benefit from transcranial magnetic stimulation? Otolaryngol Head Neck Surg 2007, 137(4):589-595.

28. Adamchic I, Tass PA, Langguth B, Hauptmann C, Koller M, Schecklmann M, Zeman F, Landgrebe M: Linking the Tinnitus questionnaire and the subjective clinical global impression: which differences are clinically important? Health Qual Life Outcomes 2012, 10:79.

29. Kleinjung T, Eichhammer $P$, Landgrebe M, Sand P, Hajak G, Steffens $T$, Strutz J, Langguth B: Combined temporal and prefrontal transcranial magnetic stimulation for tinnitus treatment: a pilot study. Otolaryngol Head Neck Surg 2008, 138(4):497-501.

30. Schecklmann M, Landgrebe $M$, Kleinjung T, Frank E, Rupprecht $R$, Sand PG, Eichhammer P, Hajak G, Langguth B: State- and trait-related alterations of motor cortex excitability in tinnitus patients. PLoS One 2014, 9(1):e85015.

31. Bajbouj M, Lisanby SH, Lang UE, Danker-Hopfe H, Heuser I, Neu P: Evidence for impaired cortical inhibition in patients with unipolar major depression. Biol Psychiatry 2006, 59(5):395-400.

32. Chistyakov AV, Kaplan B, Rubichek O, Kreinin I, Koren D, Feinsod M, Klein E: Antidepressant effects of different schedules of repetitive transcranial magnetic stimulation vs. clomipramine in patients with major depression: relationship to changes in cortical excitability. Int $J$ Neuropsychopharmacol 2005, 8(2):223-233.

33. Dolberg OT, Dannon PN, Schreiber S, Grunhaus L: Magnetic motor threshold and response to TMS in major depressive disorder. Acta Psychiatr Scand 2002, 106(3):220-223.

34. Nahas Z, Kozel FA, Li X, Anderson B, George MS: Left prefrontal transcranial magnetic stimulation (TMS) treatment of depression in bipolar affective disorder: a pilot study of acute safety and efficacy. Bipolar Disord 2003, 5(1):40-47.

35. Triggs WJ, McCoy KJ, Greer R, Rossi F, Bowers D, Kortenkamp S, Nadeau SE, Heilman KM, Goodman WK: Effects of left frontal transcranial magnetic stimulation on depressed mood, cognition, and corticomotor threshold. Biol Psychiatry 1999, 45(11):1440-1446.

36. Mantovani A, Lisanby SH, Pieraccini F, Ulivelli M, Castrogiovanni P, Rossi S: Repetitive Transcranial Magnetic Stimulation (rTMS) in the treatment of panic disorder (PD) with comorbid major depression. J Affect Disord 2007, 102(1-3):277-280.

37. Ahmed MA, Darwish ES, Khedr EM, El Serogy YM, Ali AM: Effects of low versus high frequencies of repetitive transcranial magnetic stimulation on cognitive function and cortical excitability in Alzheimer's dementia. J Neurol 2012, 259(1):83-92.

38. Croarkin PE, Wall CA, Nakonezny PA, Buyukdura JS, Husain MM, Sampson SM, Emslie GJ, Kozel FA: Increased cortical excitability with prefrontal high-frequency repetitive transcranial magnetic stimulation in adolescents with treatment-resistant major depressive disorder. J Child Adolesc Psychopharmacol 2012, 22(1):56-64.

39. Furukawa T, Toyokura M, Masakado Y: Suprathreshold $0.2 \mathrm{~Hz}$ repetitive transcranial magnetic stimulation (rTMS) over the prefrontal area. Tokai J Exp Clin Med 2010, 35(1):29-33.

40. Pallanti S, Di Rollo A, Antonini S, Cauli G, Hollander E, Quercioli L: Lowfrequency rTMS over right dorsolateral prefrontal cortex in the treatment of resistant depression: cognitive improvement is independent from clinical response, resting motor threshold is related to clinical response. Neuropsychobiology 2012, 65(4):227-235.

41. Pretalli JB, Nicolier M, Chopard G, Vandel P, Tio G, Monnin J, Pazart L, Sechter D, Haffen E: Resting motor threshold changes and clinical response to prefrontal repetitive transcranial magnetic stimulation in depressed patients. Psychiatry Clin Neurosci 2012, 66(4):344-352

42. Spampinato C, Aguglia E, Concerto C, Pennisi M, Lanza G, Bella R, Cantone M, Pennisi G, Kavasidis I, Giordano D: Transcranial magnetic stimulation in the assessment of motor cortex excitability and treatment of drug-resistant major depression. IEEE Trans Neural Syst Rehabil Eng 2013, 21(3):391-403.

43. Cuypers K, Thijs H, Meesen RL: Optimization of the transcranial magnetic stimulation protocol by defining a reliable estimate for corticospinal excitability. PLoS One 2014, 9(1):e86380.
44. Bavelier D, Neville HJ: Cross-modal plasticity: where and how? Nat Rev Neurosci 2002, 3(6):443-452.

45. Merabet LB, Pascual-Leone A: Neural reorganization following sensory loss: the opportunity of change. Nat Rev Neurosci 2010, 11(1):44-52.

46. Rothen N, Terhune DB: Increased resting state network connectivity in synesthesia: evidence for a neural basis of synesthetic consistency. J Neurosci 2012, 32(40):13641-13643.

47. Dovern A, Fink GR, Fromme AC, Wohlschlager AM, Weiss PH, Riedl V: Intrinsic network connectivity reflects consistency of synesthetic experiences. J Neurosci 2012, 32(22):7614-7621.

48. Llinas RR, Ribary U, Jeanmonod D, Kronberg E, Mitra PP: Thalamocortical dysrhythmia: A neurological and neuropsychiatric syndrome characterized by magnetoencephalography. Proc Natl Acad Sci U S A 1999, 96(26):15222-15227.

49. Salvi R, Lobarinas E, Sun W: Pharmacological Treatments for Tinnitus: New and Old. Drugs Future 2009, 34(5):381-400.

50. Ziemann U: TMS and drugs. Clin Neurophysiol 2004, 115(8):1717-1729.

51. Ziemann U, Meintzschel F, Korchounov A, llic TV: Pharmacological modulation of plasticity in the human motor cortex. Neurorehabil Neural Repair 2006, 20(2):243-251

52. Di Lazzaro V, Pilato F, Dileone M, Ranieri F, Ricci $V$, Profice P, Bria P, Tonali PA, Ziemann U: GABAA receptor subtype specific enhancement of inhibition in human motor cortex. J Physiol 2006, 575(Pt 3):721-726.

53. Yang S, Weiner BD, Zhang LS, Cho SJ, Bao S: Homeostatic plasticity drives tinnitus perception in an animal model. Proc Natl Acad Sci U S A 2011, 108(36):14974-14979.

54. Sand PG, Langguth B, Itzhacki J, Bauer A, Geis S, Cardenas-Conejo ZE, Pimentel $V$, Kleinjung $T$ : Resequencing of the auxiliary GABA(B) receptor subunit gene KCTD12 in chronic tinnitus. Front Syst Neurosci 2012, 6:41.

55. Wu ZM, Chen ML, Wu XH, Li L: Interaction between auditory and motor systems in speech perception. Neurosci Bull 2014, 30(3):490-496.

56. Honing $\mathrm{H}$ : Without it no music: beat induction as a fundamental musical trait. Ann N Y Acad Sci 2012, 1252:85-91.

57. Pineda JA: The functional significance of mu rhythms: translating "seeing" and "hearing" into "doing". Brain Res Brain Res Rev 2005, 50(1):57-68.

doi:10.1186/1471-2202-15-71

Cite this article as: Schecklmann et al.: Changes in motor cortex excitability associated with temporal repetitive transcranial magnetic stimulation in tinnitus: hints for cross-modal plasticity? BMC Neuroscience 2014 15:71

\section{Submit your next manuscript to BioMed Central and take full advantage of:}

- Convenient online submission

- Thorough peer review

- No space constraints or color figure charges

- Immediate publication on acceptance

- Inclusion in PubMed, CAS, Scopus and Google Scholar

- Research which is freely available for redistribution 